\title{
FDA's New Guidance for Industry Addressing Evaluation of the Safety of New Drugs for Improving Glycemic Control: A Case Study in Regulatory Science
}

\author{
J. Rick Turner ${ }^{1}$
}

Published online: 21 October 2020

(c) The Drug Information Association, Inc 2020

On March 9, 2020, the US Food and Drug Administration (FDA) issued a draft guidance entitled "Type 2 Diabetes Mellitus: Evaluating the Safety of New Drugs for Improving Glycemic Control" [1]. This guidance replaces two documents that are being withdrawn: the February 2008 draft guidance entitled "Diabetes Mellitus: Developing Drugs and Therapeutic Biologics for Treatment and Prevention" and the December 2008 final guidance entitled "Diabetes Mellitus-Evaluating Cardiovascular Risk in New Antidiabetic Therapies to treat Type 2 Diabetes", referred to from now on as the 2008 Cardiovascular Risk Guidance. This Editorial first discusses the genesis, content, and consequences of 2008 Cardiovascular Risk Guidance, and then describes the content of the new March 2020 guidance. Finally, it places multiple events and activities of academicians, clinical researchers, physician scientists, drug developers, and regulators between May 2007 and March 2020 into a case study in regulatory science.

\section{Genesis of the 2008 Evaluating Cardiovascular Risk Guidance}

The challenges of diabetes to long-term health are enormous. The life expectancy of a patient with type 2 diabetes can be reduced considerably, driven to a large extent by increased risk of heart disease, stroke, and renal disease. Quality as well as quantity of life can also be seriously affected given the additional burdens of nervous system damage, blindness, and lower limb amputation [2]. When first-line interventions including dietary modification and increased physical

\footnotetext{
J. Rick Turner

j.rick.turner123@outlook.com

1 Department of Pharmacy Practice, Campbell University College of Pharmacy \& Health Sciences, 239 J.P. Riddle Building, PO Box 1090, Buies Creek, NC 27506, USA
}

activity have failed to halt the disease's progression, prescription of pharmaceutical agents becomes necessary (lifestyle interventions should be maintained). While many classes of antidiabetic drugs have been approved in the US for adults with type 2 diabetes, the continuing medical need for additional pharmaceutical agents is well captured by the European Medicines Agency's guideline addressing clinical investigation of medicinal products in the treatment or prevention of diabetes, which comments as follows: "Glucose control in type 2 diabetes deteriorates progressively over time, and, after failure of diet and exercise alone, needs on average a new intervention with glucose-lowering agents every 3-4 years in order to obtain/retain good control" [3].

There are multiple aspects of bringing a new drug for type 2 diabetes to market, including safety investigations. In May 2007, the New England Journal of Medicine e-published a meta-analysis purporting to show an increased cardiovascular risk associated with the thiazolidinedione drug rosiglitazone [4], which had been approved by FDA in 1999. One result from the meta-analysis was an odds ratio for myocardial infarction in the rosiglitazone group compared with the control group of 1.43 (95\% CI 1.03 to $1.98, p=0.03$ ). This result can be interpreted as follows: it is compatible with an increase in risk of myocardial infarction of as little as 3\% and as great at $98 \%$, and the best estimate is an increase of $43 \%$.

While many researchers and physicians criticized the methodology used in the meta-analysis and the authors' interpretations its results [5-7], reactions to its publication precipitated a cascade of events, including a joint meeting of FDA's Endocrinologic and Metabolic Drugs Advisory Committee and its Drug Safety and Risk Management Committee in July 2007 to discuss the cardiovascular ischemic and thrombotic risk of the thiazolidinediones, with a focus on rosiglitazone. (Full disclosure: I was an invited speaker at the Open Public Hearing session at this meeting: my testimony was that the meta-analysis had been so poorly conducted that no decision-making weight should be afforded 
it.) In line with the committees' members' $22-1$ vote that rosiglitazone should not be removed from the market, FDA did not remove it. However, in an abundance of cautionpersons with diabetes are at higher risk for cardiovascular disease-in November 2008 FDA released the 2008 Cardiovascular Risk Guidance.

\section{Content of the 2008 Cardiovascular Risk Guidance}

Prior to submission of a New Drug Application (NDA) or Biologics License Application (BLA), sponsors are required to compare the incidence of centrally adjudicated major adverse cardiovascular events (MACE) composite endpoint outcomes (typically nonfatal myocardial infarction, nonfatal stroke, and cardiovascular death) occurring in the drug treatment arm with those occurring in the control treatment arm to show that the upper bound of the two-sided $95 \%$ confidence interval for the risk ratio point estimate is less than 1.8. In more generally accessible language, this requirement translates to the prospective exclusion of excess cardiovascular risk of $80 \%$ or greater.

This is the first step in what is usually a two-stage process. The requirement can be satisfied by performing a metaanalysis of adjudicated cardiovascular events using participant level data from the phase 2 and phase 3 trials conducted in the drug's clinical development program, or, if the data from the studies included in the meta-analysis will not meet this requirement, by conducting a large cardiovascular safety outcome trial that, alone or when added to other the trials, would discharge the 1.8 threshold.

If the upper bound of the two-sided 95\% confidence interval for the risk ratio point estimate is between 1.3 and 1.8 , and the overall benefit-risk analysis supports approval, a large post-marketing trial very likely is required to fulfill the second requirement, i.e., definitive demonstration that the upper bound of the two-sided 95\% confidence interval for the risk ratio point estimate is then less than 1.3. In more generally accessible language, this requirement translates to the prospective exclusion of excess cardiovascular risk of $30 \%$ or greater (see [8] for detailed discussion).

\section{Consequences of the 2008 Cardiovascular Risk Guidance}

As a result of the need to prospectively exclude an excess cardiovascular risk of $30 \%$ or greater, many large cardiovascular safety outcome trials have been conducted. For example, SAVOR-TIMI-53, EXAMINE, TECOS, and ELIXA [9-12] were among early trials to do so. The next occurrence of note was EMPA-REG OUTCOME, whose results, published in 2015, not only provided evidence of the absence of an unacceptable increase in cardiovascular risk associated with empagliflozin but also provided evidence of a cardiovascular protective effect [13]. Subsequently, in December 2016, FDA approved a new indication for empagliflozin to reduce the risk of cardiovascular death in adult patients with Type 2 diabetes and established cardiovascular disease [14], the first occurrence of FDA granting an indication of cardiovascular benefit for an antidiabetic drug for Type 2 diabetes. Other trials, including LEADER and SUSTAIN-6 [15, 16], have reported various types and degrees of cardiovascular benefit associated with antidiabetic agents (see $[17,18]$ for extended discussions).

Other publications of interest in the literature include those discussing the potential therapeutic renal effects of antidiabetic agents $[19,20]$ and one suggesting revisiting the 2008 Cardiovascular Risk Guidance [21].

\section{Evolution of FDA's Views Regarding the Cardiovascular Safety of Rosiglitazone}

Another joint meeting of FDA's Endocrinologic and Metabolic Drugs Advisory Committee and its Drug Safety and Risk Management Advisory Committee was held in July 2010. On this occasion, the committee members voted 20-12 against removing the rosiglitazone from the market. However, of the 20 members voting against marketing withdrawal, 10 voted for additional warnings and restrictions on use of the drug and seven voted for additional warnings. Subsequent to the meeting, while rosiglitazone was not withdrawn from the market, the drug's sponsor was required to submit a Risk Evaluation and Mitigation Strategy (REMS) within 60 days of the Agency's announcement of this decision on September 23, 2010 [22].

In 2013, Mahaffey and colleagues [23] published a paper presenting the results of a reevaluation of cardiovascular endpoints in the sponsor's Rosiglitazone Evaluated for Cardiac Outcomes and Regulation of Glycaemia in Diabetes (RECORD) trial that had been requested by FDA. Following their review of these results, in November 2013 FDA announced their substantially reduced concern about the risk of myocardial infarction associated with rosiglitazone and removed the REMS's restrictions on prescribing and dispensing rosiglitazone-containing drugs. In December 2015, FDA went further by eliminating the REMS altogether, stating in a drug safety communication that it was no longer necessary to ensure that the therapeutic benefits of rosiglitazone-containing drugs outweighed their risks.

Given that announcement, it can be reasonably argued that the FDA has thought from that time that the 2007 metaanalysis [4] that led to events discussed in this Editorial did 
not accurately represent the true safety characteristics of rosiglitazone with regard to myocardial infarction.

\section{Summary of the 2020 Draft Guidance's Content}

As noted in its introduction, "This guidance provides recommendations on the size and nature of the safety databases needed to support drugs for chronic use to improve glycemic control in patients with type 2 diabetes ... [it] is intended to serve as a focus for continued discussions among the Division of Metabolism and Endocrinology Products, pharmaceutical sponsors, the academic community, and the public" [1]. Its content was driven by discussions at a meeting of the FDA's Endocrinologic and Metabolic Drugs Advisory Committee in October 2018 [24] at which members were asked to consider three points: the robustness of safety bases before the December 2008 Cardiovascular Risk Guidance; new information gained from considering the multiple cardiovascular outcome safety trials conducted since that guidance's release; and whether that guidance's recommendations were still appropriate. Based on the discussions, a new approach to the evaluation of a new drug intended to improve glycemic control in patients with type 2 diabetes is presented.

\section{Size of the New Drug's Safety Database}

When submitting a marketing application, a new drug's safety database should include data from controlled clinical trials and controlled clinical trial extensions with these exposures:

1. At least 4000 participant-years of exposure to the drug in phase 3 clinical trials (including all doses studied in the trials)

2. At least 1500 participants exposed to the drug for at least 1 year

3. At least 500 participants exposed to the drug for at least 2 years.

\section{Participant Characteristics}

Comorbidities and diabetes-related complications such as cardiovascular and chronic kidney disease are common in patients with type 2 diabetes. It is therefore important to evaluate a new drug's safety in patients who will be prescribed the drug, including those with cardiovascular and chronic kidney disease, and older patients. Therefore, the safety database should include the following in phase 3 trials:
1. At least 500 participants with stage $3 / 4$ chronic kidney disease exposed to the drug

2. At least 600 participants with established cardiovascular disease (e.g., previous myocardial infarction, documented coronary artery disease, previous stroke, and peripheral vascular disease) exposed to the drug

3. At least 600 participants older than 65 years of age exposed to the drug.

Recognizing that a given participant could fall into more than one of these three categories, sponsors should aim for at least 1200 participants with at least one of these conditions [1].

\section{Other Considerations}

Sponsors should also account for these considerations concerning the collection of safety data:

1. Adverse cardiovascular outcomes remain an important source of morbidity and mortality for patients with type 2 diabetes mellitus. Therefore, sponsors should use rigorous methods for the collection of adverse cardiovascular events, and asses them by adjudication.

2. In some cases, the evaluation of a premarketing safety concern may require that a drug development program accrue a minimum number of relevant adverse events to exclude a meaningful degree of risk. Adjudication of these adverse events may also be needed. FDA expects that situations where the collection of these additional safety data is necessary will be identified and discussions held between the sponsor and FDA before initiation of phase 3 trials.

3. Sponsors should include data safety monitoring boards or committees to provide independent oversight of clinical trials' safety findings.

\section{An Associated FDA News Release}

In an associated news release dated March 9, 2020 [25], FDA noted that the new guidance addresses broader safety evaluations for new drugs for type 2 diabetes beyond cardiovascular outcome trials.

\section{Submitting Comments on the Draft Guidance}

As is the case for all draft guidances issued by FDA, for a certain time after their release (in this case until June 8, 2020), any interested party may submit comments to the FDA docket associated with the guidance, accessed via 
an announcement in the Federal Register [26]. Submitting comments by this date will ensure that FDA considers them before it begins work on the final version of the guidance. All submitted comments will be visible on the docket. Additionally, if you are reading this Editorial after June 8, 2020, comments can be submitted to FDA at any time (as for all guidances) in accordance with FDA's good guidance practices.

The announcement in the Federal Register [26] noted that FDA is interested in answers to the following questions/ topics:

- Is it more important to emphasize the number of participants exposed to the drug or the amount of exposure (i.e., number of participant-years), or should expectations be set for both of these parameters?

- What would constitute a minimally acceptable database (either in terms of the number of participants, the number of participant-years, or both) regarding exposure to the drug at the time of submitting a marketing application?

- What comorbid conditions are important to include, and what would be a minimally acceptable number of participants or participant-years to include for each of them?

- Are there specific safety concerns for patients with type 2 diabetes that should be rigorously evaluated, and if so, how should those evaluations be conducted?

- Is the adjudication of adverse events related to a specific safety concern a necessary part of the safety evaluation, and if so, should it be conducted by an independent, blinded adjudication committee or would other means of adjudication be adequate?

\section{A Case Study in Regulatory Science}

Regulatory science is "the science of developing new tools, standards, and approaches to assess the safety, efficacy, quality, and performance of all FDA-regulated products" [27]. Many stakeholders play major roles in advancing regulatory science, including regulators, experts from academia and industry, and, increasingly, patients. The results of collaborations between these groups can be particularly significant $[8,21,24]$.

As discussed earlier, for each new antidiabetic drug for type 2 diabetes, the 2008 Cardiovascular Risk Guidance requires the prospective exclusion of excess cardiovascular risk of $30 \%$ or greater, which was typically done by conducting a large cardiovascular outcome study. None of these studies conducted between the release of the 2008 Cardiovascular Risk Guidance and the release of the new guidance in March 2020 has identified an increased cardiovascular risk, and some have shown cardiovascular therapeutic benefit. As noted in the Federal Register, "The FDA continues to review new clinical trial evidence and update its recommendations for drug development to reflect emerging scientific information", and, accordingly, FDA is withdrawing that guidance "because its recommendations for safety assessment have become outdated" [26].

It will be of great interest to many of us to see the content of the final version of this new guidance when it is released, and to follow future investigations of the safety of new drugs for improving glycemic control resulting from its recommendations.

\section{References}

1. FDA. Guidance for Industry. Type 2 diabetes mellitus: evaluating the safety of new drugs for improving glycemic control. Draft; March 2020. Available at https://www.fda.gov/media/135936/ download Accessed 15 April 2020.

2. Turner JR, Karnad DR, Kothari S. Cardiovascular safety in drug development and therapeutic use: new methodologies and evolving regulatory landscapes. Gewerbestrasse: Springer International Publishing; 2017.

3. EMA. Guideline on the clinical investigation of medicinal products in the treatment or prevention of diabetes mellitus. Effective November 2012. Available at: http://www.ema.europa.eu/docs/ en_GB/document_library/Scientific_guideline/2012/06/WC500 129256.pdf (Accessed April 17, 2020).

4. Nissen SE, Wolski K. Effect of rosiglitazone on the risk of myocardial infarction and death from cardiovascular causes. N Engl J Med. 2007;356:2457-71 Epub 2007 May 21.

5. Diamond GA, Bax L, Kaul S. Uncertain effects of rosiglitazone on the risk for myocardial infarction and cardiovascular death. Ann Intern Med. 2007;147:578-81.

6. Pignone M. Rosiglitazone appears to be associated with an increased risk of cardiovascular events. Clin Diabetes. 2007;25:123-4.

7. Turner JR, Durham TA. Integrated cardiac safety: assessment methodologies for noncardiac drugs in discovery, development, and postmarketing surveillance. Hoboken: Wiley; 2009.

8. Geiger MJ, Mehta C, Turner JR, et al. Clinical development approaches and statistical methodologies to prospectively assess the cardiovascular risk of new antidiabetic therapies for type 2 diabetes. Ther Innov Regul Sci. 2015;49:50-64.

9. Scirica BM, Bhatt DL, Braunwald E, et al. SAVOR-TIMI 53 Steering Committee and Investigators. Saxagliptin and cardiovascular outcomes in patients with type 2 diabetes mellitus. N Engl J Med. 2013;369:1317-26.

10. White WB, Cannon CP, Heller SR, ELIXA Investigators, et al. Alogliptin after acute coronary syndrome in patients with type 2 diabetes. N Engl J Med. 2013;369:1327-35.

11. Green JB, Bethel MA, Armstrong PW, TECOS Study Group, et al. Effect of sitagliptin on cardiovascular outcomes in type 2 diabetes. N Engl J Med. 2015;373:232-42.

12. Pfeffer MA, Claggett B, Diaz R, ELIXA Investigators, et al. Lixisenatide in patients with type 2 diabetes and acute coronary syndrome. N Engl J Med. 2015;373:2247-57.

13. Zinman B, Wanner C, Lachin JM, EMPA-REG OUTCOME Investigators, et al. Empagliflozin, cardiovascular outcomes, and mortality in type 2 diabetes. N Engl J Med. 2015;2015(373):2117-28.

14. FDA News Release. FDA approves Jardiance to reduce cardiovascular death in adults with type 2 diabetes. Available at: https ://www.fda.gov/news-events/press-announcements/fda-appro 
ves-jardiance-reduce-cardiovascular-death-adults-type-2-diabe tes. Accessed 18 April 2020.

15. Marso SP, Daniels GH, Brown-Frandsen K, LEADER Steering Committee; LEADER Trial Investigators, et al. Liraglutide and cardiovascular outcomes in type 2 diabetes. N Engl J Med. 2016;375:311-22.

16. Marso SP, Bain SC, Consoli A, SUSTAIN-6 Investigators, et al. Semaglutide and cardiovascular outcomes in patients with type 2 diabetes. N Engl J Med. 2016;375:1834-44.

17. Turner JR. Integrated cardiovascular safety: multifaceted considerations in drug development and therapeutic use. Expert Opin Drug Saf. 2017;16:481-92.

18. Yandrapalli S, Jolly G, Horblitt A, et al. Cardiovascular safety and benefits of non-insulin antihyperglycemic drugs for the treatment of type 2 diabetes mellitus-part 1. Cardiol Rev. 2020 Mar 19 [Epub ahead of print].

19. Chu C, Lu YP, Yin L, Hocher B. The SGLT2 inhibitor empagliflozin might be a new approach for the prevention of acute kidney injury. Kidney Blood Press Res. 2019;44:149-57.

20. Zelniker TA, Wiviott SD, Raz I, et al. SGLT2 inhibitors for primary and secondary prevention of cardiovascular and renal outcomes in type 2 diabetes: a systematic review and meta-analysis of cardiovascular outcome trials. Lancet. 2019;393(10166):31-9.

21. Sharma A, Pagidipati NJ, Califf RM, et al. Impact of regulatory guidance on evaluating cardiovascular risk of new glucose-lowering therapies to treat type 2 diabetes mellitus: lessons learned and future directions. Circulation. 2020;141:843-62.
22. Woodcock J, Sharfstein JM, Hamburg M. Regulatory action on rosiglitazone by the US Food and Drug Administration. N Engl J Med. 2010;363:1489-91.

23. Mahaffey KW, Hafley G, Dickerson S, et al. Results of a reevaluation of cardiovascular outcomes in the RECORD trial. Am Heart J. 2013;166(240-249):e1.

24. FDA. Summary Minutes of the Endocrinologic and Metabolic Drugs Advisory Committee Meeting, October 24-25, 2018. Available at: https://www.fda.gov/media/121265/download. Accessed 18 April 2020.

25. FDA News Release. March 9, 2020. FDA proposes broad approach for conducting safety trials for type 2 diabetes medications. Available at: https://www.fda.gov/news-events/press-announcements/ fda-proposes-broad-approach-conducting-safety-trials-type-2-diabetes-medications. Accessed 19 April 2020.

26. Federal Register. March 10, 2020. Type 2 diabetes mellitus: evaluating the safety of new drugs for improving glycemic control; Draft guidance for industry; Notice of Availability. Available at: https://www.federalregister.gov/documents/2020/03/10/202004877/type-2-diabetes-mellitus-evaluating-the-safety-of-newdrugs-for-improving-glycemic-control-draft. Accessed 19 April 2020.

27. FDA. Advancing Regulatory Science. Available at: https://www. fda.gov/science-research/science-and-research-special-topics/ advancing-regulatory-science. Accessed 20 April 2020. 